\title{
EDITORIAL
}

\section{Neonatal abstinence syndrome: a policy perspective}

\author{
Journal of Perinatology (2015) 35, 539-541. doi:10.1038/jp.2015.53
}

Neonatal abstinence syndrome (NAS) was first described in the 1970s, identified among neonates whose mothers most commonly used heroin or were on methadone maintenance. ${ }^{1}$ NAS refers to the collection of signs and symptoms that occur when a newborn prenatally exposed to opiates experiences opioid withdrawal. This syndrome is primarily characterized by irritability, tremors, feeding problems, vomiting, diarrhea, sweating and, in some cases, seizures. ${ }^{2}$ However, not all prenatally exposed infants develop signs of abstinence, for maternal or infant factors, most of which are not understood at this point, can affect the expression of NAS.

Documenting the nature and severity of the problem is complicated by the use of a wide variety of instruments and approaches $^{3}$ for diagnosing NAS and quantifying the severity of neonatal abstinence signs and symptoms. The most commonly used NAS assessment tool in the United States, the Modified Finnegan's Neonatal Abstinence Scoring Tool, can be characterized by poor inter-rater reliability, ${ }^{4}$ especially regarding neurologic sequelae, and, without proper and thorough training, it can be affected by administrator bias. $^{5}$

Over the past several decades, the incidence of NAS has varied, usually associated with the waxing and waning of heroin use in the general population. However, an increase in the use and misuse of prescription narcotic medications has emerged over the past several years, ${ }^{6}$ coinciding with the increased rates of NAS documented in the article by Patrick et al in this month's Journal of Perinatology. It is important to note, however, that the current rise in the number of infants with NAS represents a wide spectrum of pregnant women's use of opiates. These uses include heroin addiction, polydrug use, sanctioned and nonsanctioned prescription opioid use and medication (methadone or buprenorphine)assisted treatment (MAT). ${ }^{7}$

The unique nature of the current upswing in the occurrence of NAS is well characterized in a report from the Tennessee Department of Health. ${ }^{8}$ Through its web-based reporting portal, data on the source of maternal opiate use in 805 reported cases of NAS revealed that $46 \%$ of cases of NAS resulted from the mother's being on supervised MAT, 39\% were due to abuse of prescribed opioids and $19 \%$ were due to supervised pain therapy. Illicit use of opiates without a prescription accounted for only $28 \%$ of the NAS cases. (The percentage total is over $100 \%$ because of high rates of polydrug use in the population.) In a study of prescription narcotic use by pregnant women for medically indicated reasons, the use of narcotic prescriptions for at least one month during pregnancy increased from about 2.5 cases per 1000 deliveries to more than 10 cases per 1000 deliveries between 1998 and 2008. ${ }^{9}$ From these two sets of data, it can be surmised that a significant number of cases of NAS are occurring in infants whose mothers have been prescribed opiates during the pregnancy.

This calls into question the appropriate prescribing and dosing of opiate medications for pain therapy during pregnancy, as well as MAT, such as with the prescribed use of methadone, which has been shown to significantly improve pregnancy outcome among heroin-using women. ${ }^{10}$ As compared with the ongoing use of street heroin, pregnant women enrolled in medication-assisted treatment programs have lower rates of preterm labor, intrauterine growth retardation and fetal and maternal morbidity and mortality, to name a few advantages. However, little research has been done to document the advantages of MAT among women who are using prescription opiates, either legally or illegally.

States have a vested interest in addressing these issues. Vermont, for example, covers more than $90 \%$ of deliveries to women with opioid dependency through its Medicaid program. ${ }^{11}$ The 2009 Substance Abuse and Mental Health Services Administration's report, 'Substance-Exposed Infants: State Responses to the Problem,' provides a five-point intervention framework to organize prevention and intervention opportunities that can affect outcomes for opioid-dependent women and their children. ${ }^{12}$ States are in a position to build on this model and develop an even broader systems perspective that stretches from the preconceptional period through childhood and addresses clinical and systems issues across multiple domains.

\section{Preconceptional}

1. Professional education: provide education on the impact of prescription and nonprescription opiate use and abuse on pregnancy and neonatal outcome, validated screening and brief intervention approaches to identify women at risk for substance use and abuse and the role of and guidelines for MAT for pregnant women who are using or abusing opiates.

2. Public health campaign: educate the public regarding the impact of prenatal opiate use, including prescription medications and medications used for MAT, on pregnancy and the newborn.

3. Prescription surveillance: establish an integrated system for monitoring physicians prescribing opiates, pharmacies' filling of prescriptions and patient prescription records across counties and other jurisdictions within the State.

Pregnancy

1. Screening, brief intervention, referral and treatment ${ }^{13}$ : screen all pregnant women for use of alcohol, tobacco and illicit drugs via a validated screening instrument and provide an evidencebased brief intervention to all women with a positive screen. Refer women as needed to an appropriate level of treatment.

2. Record information: record all screening, brief intervention, referral and treatment data in the patient's obstetric chart.

3. Treatment: identify service delivery issues for the provision of treatment for pregnant women, including MAT with collaboration and coordination between methadone and buprenorphine treatment programs and prenatal care providers.

4. Information transfer: establish Health Insurance Portability and Accountability Act-consistent policies within hospitals to ensure that the child's pediatrician and other providers in the neonatal nursery have access to prenatal records so as to know of possible prenatal opiate exposure.

\section{Newborn care}

1. Assessment for neonatal abstinence: use a validated NAS assessment tool to monitor the newborn's signs and symptoms related to opiate withdrawal. 
a. Ensure that all physicians and nursing personnel are thoroughly trained on the assignment of scores on the NAS tool.

b. Include training on the use of the abstinence score in orientation for all new nurses and physicians in the neonatal intensive care unit, mother/baby unit, pediatric unit and pediatric intensive care unit.

c. Monitor inter-rater reliability within an institution on at least an annual basis.

2. NAS therapy: develop institutional guidelines for a consistent, evidence-based approach to supportive and pharmacologic interventions for the newborn affected by prenatal opiate exposure.

3. CAPTA: the Child Abuse Prevention and Treatment Act (CAPTA) requires reporting of all children at birth to the child welfare system if they are affected by prenatal exposure to alcohol or illicit drugs. Ensure that all healthcare and child welfare personnel understand the intent and process for CAPTA implementation in the State and that procedures are established for linking reported infants to early intervention programs.

4. Child welfare: develop a plan of safe care for the infant and for ongoing treatment to support the birth mother.

\section{Pediatric care}

Follow-up: provide ongoing developmental and behavioral screening and oversight for the child, ensuring access to earliest interventions as needed. In the majority of cases, these services can be covered through federal Early Periodic Screening, Diagnosis and Treatment funds. Strengthen collaborations between clinical providers, community agencies, home visiting programs and state agencies to track substance-exposed infants and their families through early childhood.

\section{State systems}

1. Data collection: collect and track data on the number of cases and outcomes of substance-exposed infants and NAS. Incorporate child welfare data into this tracking system. In addition, establish a reporting system for NAS that can determine the source of the pregnant woman's access to opiates.

2. Reimbursement: provide medicaid and commercial insurance reimbursement for using screening protocols that detect substance abuse early in pregnancy and developmental risk in exposed infants and children.

3. Quality of care: use collaborative efforts to strengthen clinical standards for identification, management and follow-up with NAS-affected infants and their families.

4. Access to substance abuse treatment: develop oversight measures to ensure follow-up with opioid-dependent women and receipt of comprehensive services during pregnancy and after. As one component of this effort, ensure that state policies on substance use and abuse in pregnancy operate within the context of a public health approach rather than within a criminal or punitive framework.

Federal policies

1. Oversight: federal agencies have oversight roles for state and local agencies that could directly affect NAS and related outcomes, especially through the data collection functions carried out at a national level. Oversight of the CAPTA legislation mentioned previously, the review of national trends in admissions of pregnant women and review of public and private coverage for treatment for mothers and infants would help practitioners know more about the options for responding to NAS. This should also involve placing NAS/opioid issues in a broader context, recognizing that only one out of ten persons who need substance abuse treatment receive treatment and only $1.2 \%$ of all admissions to publicly funded treatment programs in 2011 were pregnant women. ${ }^{14}$ Federal data sets, including the National Survey of Drug Use and Health ${ }^{15}$ and the Treatment Episode Data Set, ${ }^{16}$ can provide useful context for states and for policy makers.

2. Standards for screening and treatment: federal monitoring of insurers and health providers under both Medicaid and commercial insurance coverage could enhance access to treatment and reduce the tendency of some insurers and managed care firms to shorten treatment below clinically indicated levels. Funding prenatal services and births without screening for substance exposure misses a critical opportunity to identify needs and intervene early, rather than paying much more to respond later in a child's life.

3. Coordinated efforts: the recent Government Accountability Office (GAO) report (GAO-15-203) ${ }^{17}$ reviewing federal interagency coordination efforts related to opioid use in pregnancy and NAS called for stronger coordinated efforts among federal agencies. Addressing the CAPTA reporting issues mentioned above and responding to the clinical needs of infants with NAS ideally involves providers funded by federal, state and local agencies that oversee treatment, child welfare, maternal and child health, family income and early childhood services. The coordinative tasks are many, as the GAO report noted, but they ultimately affect much of what happens, or does not happen, in direct interactions between health and human services providers and children and their families.

NAS is a critical and growing problem. However, concern for prenatal exposure as it affects infants and children leads logically to the question of what other forms of prenatal exposure have detrimental effects and at what scale. For all its recent growth, NAS remains a small segment in the overall picture related to the harmful effects of legal and illegal substance use, notably alcohol and tobacco, during pregnancy. Ultimately, state and federal policy makers and healthcare clinicians must address the larger context of substance-exposed infants rather than focusing on one substance at a time.

\section{CONFLICT OF INTEREST}

The authors declare no conflict of interest.

$$
\begin{array}{r}
\text { IJ Chasnoff }{ }^{1,2} \text { and S Gardner } \\
{ }^{3} \text { Department of Pediatrics, University of Illinois College } \\
\text { of Medicine, Chicago, IL, USA; } \\
{ }^{2} \mathrm{NTI} \text { Upstream, Chicago, IL, USA and } \\
{ }^{3} \text { Children and Family Futures, Lake Forest, CA, USA } \\
\text { E-mail: irachasnoff@gmail.com }
\end{array}
$$

\section{REFERENCES}

1 Finnegan LP, Kron RE, Connaughton JF, JP E. Assessment and treatment of abstinence in the infant of the drug-dependent mother. Int J Clin Pharmacol Biopharm 1975; 12(1-2): 19-32.

2 Hudak M, Tan R. The Committee on Drugs and the Committee on Fetus and Newborn. Neonatal drug withdrawal. Pediatrics 2012; 129(2): e540-e560.

3 Crocetti MT, Amin DD, Jansson LM. Variability in the evaluation and management of opiate-exposed newborns in Maryland. Clin Pediatr 2007; 46(7): 632-635.

4 Retskin CM, Wright ME. Inter-observer reliability of the finnegan neonatal abstinence scoring tool in an acute care setting. J Obstet Gynecol Neonatal Nurs 2014 (doi:10.1111/1552-6909.12345).

5 Oei J, Lui K. Management of the newborn infant affected by maternal opiates and other drugs of dependency. J Paediatr Child Health 2007; 43(1-2): 9-18. 
6 Centers for Disease Control and Prevention. Vital signs: overdoses of prescription opioid pain relievers--United States, 1999-2008. Morb Mortal Wkly Rep 2011; 60(43): 1487-1492. Available at http://www.cdc.gov/mmwr/preview/mmwrhtml/mm6043a4. htm.

7 Patrick SW, Davis MM, Lehman CU, Cooper WO. Increasing incidence and geographic distribution of neonatal abstinence syndrome: United States 2009-2012. J Perinatol 2015; 35(8): 650-655.

8 Tennessee Department of Health. Neonatal Abstinence Syndrome Surveillance Summary for the Week of December 1-December 7, 2013. Week 49. Available at http://health.tn.gov/MCH/PDFs?NAS/NASsummary_Week_49.pdf. TennCare Office of Health Informatics. Neonatal abstinence syndrome among TennCare enrollees.

9 Kellogg A, Rose C, Harms R, Watson W. Current trends in narcotic use in pregnancy and neonatal outcomes. Am J Ob Gyn 2011; 259: e1-e4.

10 Burns L, Mattick RP, Lim K, Wallace C. Methadone in pregnancy: treatment retention and neonatal outcomes. Addiction 2007; 102: 264

11 Vermont Department of Health. Data Brief: Opioid Dependency and Pregnancy in Vermont-Medicaid Claims Data and the Vermont Uniform Hospital Discharge Data Set. Available at http://healthvermont.gov/research/documents/subab_ opi-odpreg_0113_000.pdf.

12 Young N, Gardner S, Otero C et al. Substance-exposed infants: State responses to the problem. Substance Abuse and Mental Health Services Administration. Rockville,
MD, USA. Available at http://www.ncsacw.samhsa.gov/files/Substance-Expose dlnfants.pdf.

13 Whitlock EP, Polen MR, Green CA, Orleans T, Klein J. Behavioral counseling interventions in primary care to reduce risky/harmful alcohol use by adults: a summary of the evidence for the US Preventive Services Task Force. Ann Intern Med 2004; 140: 557-568.

14 United States Department of Health and Human Services. Substance Abuse and Mental Health Services Administration. Center for Behavioral Health Statistics and Quality. Treatment Episode Data Set - Admissions (TEDS-A). 2013. Data received through 10.17.13.

15 Substance Abuse and Mental Health Services Administration. Results from the 2013 National Survey of Drug Use and Health: Summary of National Findings, Series H-48, HHS Publication no. (SMA) 14-4863. Substance Abuse and Mental Health Services Administration: Rockville, MD, USA, 2014.

16 United States Department of Health and Human Services. Substance Abuse and Mental Health Services Administration. Center for Behavioral Health Statistics and Quality. Treatment Episode Data Set - Admissions (TEDS-A). Inter-University Consortium for Political and Social Research: Ann Arbor, MI, USA, 2013.

17 United States Government Accountability Office. Report to Congressional Requesters: Prenatal drug use and newborn health - Federal efforts need better planning and coordination. 2015. Available at http://www.gao.gov/assets/670 /668385.pdf. 